\title{
A Questionnaire-based Approach on Technology Acceptance Model for Integrated Multiple Ankle Technology Device on Patient Psychology
}

\author{
Hadafi Fitri Mohd Latip ${ }^{a, c^{*}}$, A. H. Omar ${ }^{a, c}$, Tan Mei Jing ${ }^{a, c}$, Ardiyansyah Shahrom ${ }^{b, c}$ \\ ${ }^{a}$ Department of Biosciences and Health Science, Faculty of Bioscience and Medical Engineering, Universiti Teknologi Malaysia, 81310 UTM Johor Bahru, \\ Johor, Malaysia. \\ ${ }^{b}$ Faculty of Mechanical Engineering, Universiti Teknologi Malaysia, 81310 UTM Johor Bahru, Johor, Malaysia. \\ cSports Innovation and Technology Centre (SITC), Instituted Human Centered Engineering (IHCE), Universiti Teknologi Malaysia, 81310 UTM Johor Bahru, \\ Johor,Malaysia \\ *Corresponding author: hadafifitri@utm.my
}

\begin{abstract}
Sports injury is an issue that frequently occurs among athletes. The most common sports injuries are ankle sprains. Hence, to overcome this problem, the National Sports Institute and the Malaysia Sport School has set up special clinics for athletes undergoing rehabilitation treatment. In RMK 10 (2010-2015), the government aims to provide the best medical facilities and producing high value medical device. In spite of the advancement and variety of available ankle rehabilitation technology device, there is an eminent need to investigate the current position on the acceptance of these rehabilitation devices to assist patient recovery. Therefore, in this study technology acceptance model is designed to investigate the user acceptance on ankle rehabilitation technology device application in healthcare industry. The purpose of this study is to design a quantitative approach based on the technology acceptance model questionnaire as its primary research methodology. It utilized quantitative approach based on Technology Acceptance Model (TAM) to evaluate the system of ankle rehabilitation technology device. The related constructs for evaluation are: Perceived of Usefulness, Perceived Ease of Use, User Satisfaction and Attribute of Usability. All these constructs are modified to suit the context of the study. Moreover, this study outlines the details of each construct and its relevance toward the research issue. The outcome of the study represents series of approaches that will be apply to check the suitability of an ankle rehabilitation technology device on patient in healthcare industry and how well it achieve the aims and objectives of the design.
\end{abstract}

Keywords: Rehabilitation technology device, technology acceptance model, ankle, healthcare industry

(C) 2017 Penerbit UTM Press. All rights reserved

\subsection{INTRODUCTION}

Physical therapy and sport rehabilitation treatment have been, for many decades, the most standard treatment for sprained ankle. But more evidence is needed for some types of physical therapy interventions as well as rehabilitation technology device. Further investigation on cost effectiveness for the treatment of ankle sprain is also needed (Maciejasz, P. et al.,2014).

These researches combined fundamental aspects of sports science, technology and health's science to use rehabilitation technology device and an evaluation system. The research consist of three aspects; ankle training program for injury prevention and rehabilitation, FITT principle in exercise rehabilitation and Integrated Multiple Ankle Technology Device (IMATD) system.

Effective evaluation on healthcare information systems such as Integrated Multiple Ankle Technology Device (IMATD) is necessary in order to ensure systems adequately meet the requirements and information processing needs for the users and health care organizations. We designed a Technology Acceptance Model to investigate user acceptance on rehabilitation technology device in healthcare industry. It proposed a research methodology that being adopted to understand the objectives and finally able to validate the rehabilitation technology device.

Therefore, this study will describe the acceptance of the system to patients with ankle sprains as a user. The objective of this study is to identified factors contributing to the adoption of integrated Multiple Ankle Technology Device (IMATD) on ankle rehabilitation patients and to determine the level of acceptance for IMATD using Technology Acceptance Model (TAM). The acceptance of these systems is reviewed by the Technology Acceptance Model (TAM). Through this model, the patient acceptance on the system can be seen either the admission of patients to be more dominant on perception IMATD (Perceived Acceptance) or Perception Facility Acceptance (Perceived Ease of Acceptances) or other attributes.

\subsection{LITERATURE REVIEW}

TAM was developed by Davis in 1989 as one of the most widely used model in research on information technology because it is simpler and easier to implement. There are many theoretical perspectives that have been developed in order to understand how end users make decisions to use application of technology. The theories provide tools to understand the success or failure in implementation processes of 
new IT applications (Abu-Dalbouh, H. M.,2013). The most dominant theories in IT research are Innovation Diffusion Theory (IDT) (Sundaravej, T., 2010). Theory of planned Behaviour(TPB) (Ajzen, I.,1991), the Unified Theory of Acceptance and Use of Technology (UTAUT) (Venktesh et. al,2012), The FITT framework( Ammenwerth et. al,2002) and technology acceptance Model (TAM) ( Davis , 1989).

TAM model is adapted from the theory of reasoned action (TRA), the theory of reasoned action developed by the Fish and Ajzen in 1975, with the proviso that the reaction and one's perception will determine the attitude and behavior of the person. Thus, reactions will influence a user's perception on technology. TRA is a general model concerned by individual's intended behaviors. According to TRA, an individual performance is determined by the individual's attitude and norms concerning the behavior in question. In additionAjzeen and Fishbein (1980) said an individual's beliefs and motivation interact with existing behavior.

In this study the TAM theory will be used for the purpose of this study as shown in figure 1, to structure the research process and to enhance the understanding of the acceptance and use of Integrated Multiple Ankle Technology Device (IMATD) in health care industry. Individual factor such as age, gender and technology skills are external variables in the study. Perceived usefulness is assessed by means of the content and benefits of the Integrated Multiple Ankle Technology Device (IMATD) on patient progress system and the barriers and facilitators to the implementation of the system. The functionality of the application described the preciseness of the system. Attitude towards rehabilitation technology that being measured are motivation to use portable devices, satisfaction with rehabilitation technology and experience of the benefit of rehabilitation technology.

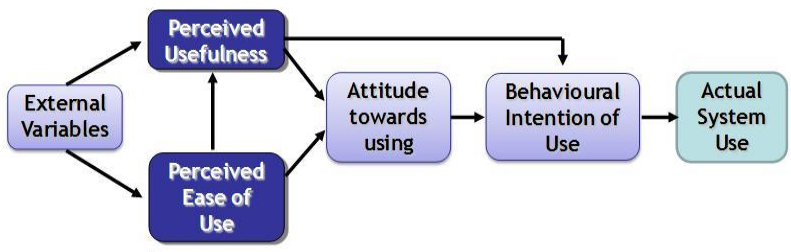

Figure 1 Technology acceptance model theory

\subsection{METHODOLOGY}

Both qualitative and quantitative method will be carried out to evaluate the effectiveness of application FITT principle in exercise rehabilitation using Integrated Multiple Ankle Technology Device (IMATD). According to Babbie (2001), the distinction between qualitative and quantitative data in social research is essentially distinct between numerical and non-numerical data. King, Keohane, and Verba (1994) stated that quantitative research use number and statistical methods. However, both methods have their own advantages and disadvantages.

According to Blaikie, N. (2007), the disadvantages of quantitative analysis is balanced by the advantages of qualitative analysis and vice versa. Each study design has different methods of data collection. Quantitative study is more towards compaction of data in order to see a bigger problem whereas the qualitative study is more towards the collection of data in detail (Tasir, Z.,2012) and a collection of empirical materials such as case study, personal experience, life story, interview, observation and historical interactional text (Denzin and Lincoln, 1994).

According to Greene and Caracelli(1997), assessing both outcomes of a study (quantitative) as well as the process (qualitative) able to showed a complete picture of the phenomenon can be developed. The combination of quantitative and qualitative studies is also known as triangulation of method (Neuman, 2000) which can increase the validity of a study.

For this reason, a questionnaire design coupled with quantitative analysis was employed in the present study to examine the variables in the adoption model and to achieve evaluation using Integrated Multiple Ankle Technology Device (IMATD) for track patient's condition. Moreover a likert scale is applied to each set of questionnaires to examine how strong the subjects agree or disagree with statements of five point scale with the following anchors:(1) Strongly disagree, (2) Disagree, (3) Nature, (4) Agree, (5) Strongly agree. In this study the proposed methodology was developed in five phases as presented in methodology section.

\subsection{Analysis Techniques}

There are three objectives of data analysis: (i) getting overview for the sample data and its attributes, (ii) testing the goodness of data and (iii) validating the proposed hypotheses.

3.2 Variable Measurement

The methodology applied in the study is based on the questionnaire approach. The objective of the questionnaire approach is basically to evaluate the Integrated Multiple Ankle Technology Device (IMATD) system. The questionnaire contained: personal information, perceived usefulness, and perceived ease of use, user satisfaction and attribute of usability. All of these have a number of questions constructed to evaluate the effectiveness of the system to the intended users

\subsection{Research Method}

This study focused on the views and opinions of ankle sprain patient's using ankle rehabilitation device technology. This study developed the questionnaire as a research tool to collect data. The questionnaire used is designed and developed based on Technology Acceptance Model (TAM). The survey is divided into 3 parts, the first part is the information respondents, the second part is a set of questionnaire on patient with ankle sprain and the last part is a questionnaire on the appliance of ankle rehabilitation technology device based on Technology Acceptance Model (TAM). Likert scale was used in the questionnaire with scale of 1 to 5 as a measurement scale for the respondents to answer based on the questions raised. . 


\section{Phase 1: Research Background}

In this phase, the researcher is required to prepare background of the problem to indicate the significance of the research. Then, followed by thorough literature review to provide evidences and supports on the research works. The researcher then developed the objectives of the research which will guide the researcher to conduct the research work and have its own independent methodology.

\section{Phase 2: Observation and Documentation}

In this phase, observation and documentation were carried out to identify conventional treatment in exercise rehabilitation and application of FITT principle in exercise rehabilitation. In this phase, observation was carried out at National Sport Council Institute under rehabilitation department to determine the application of FITT principle in exercise rehabilitation and to gather data on patients with ankle sprain from 2010 until 2015 at Sekolah Sukan Tunku Mahkota Ismail. Then, the researcher is required to identify the type of therapeutic exercise suitable for athletes.

\section{Phase 3: Design Exercise Rehabilitation Protocol}

The protocol development phase focused on developing an upgrade model of exercise rehabilitation treatment with the combination of FITT principle. This protocol is developed based on the results obtained from an observation and documentation in phase 2 .

Phase 4: Design and development Integrated Multiple Ankle Technology device (IMATD).

The development of the system in this phase emphasized on the design and development of Integrated Multiple Ankle Technology Device (IMATD). The IMATD device functioned as an information management that will be used by physical therapist in Hospital and as a medium to provide feedback to patients. The device is developed based on a system development lifecycle model (SDLC), namely the ADDIE model.

\section{Phase 5: Evaluation with Integrated Multiple Ankle Technology Device (IMATD)}

This phase is all about the findings from the research. Reliability and validity test is conducted in this phase to ensure the effectiveness of IMATD so it can be use without any error in future. The evaluation is performed to determine the correctness of the IMATD system. It utilized a quantitative approach based a Technology Acceptance Model (TAM).

\section{Phase 6: Finding, Result, Documentation and Report Writing}

This phase documented the research work starting from research background to research methodology. Flow chart is represented to illustrate the flow of the research. Scientific analysis regarding the findings of each of the objective is presented in the documentation.

\subsection{RESULTS AND DISCUSSION}

The standard software categorizes quality into functionality, Perceived of Usefulness, Perceived Ease of Use, User satisfaction and attribute of usability. This study aims to design all these categories to investigate the user acceptance on rehabilitation technology device towards patients' progress in health care industry. From the perspective of TAM, perceived ease of use, perceived usefulness, user satisfaction and attribute of usability are assumed to be related to the acceptance of rehabilitation technology device in tracking patient progress.

\section{Perceived Of Usefulness}

It is defined as the degree to which a healthcare professional believes that healthcare industry will be improving by using rehabilitation technology in tracking patient condition. The measurement of perceived usefulness comprises of 5 items modified to the content of this study as shown in Table 1.

\section{Perceived Ease of Use}

It referred to the degree to which user believed the use of rehabilitation technology device to improve the quality of treatment in the hospitals. The measurement of perceived ease of use construct contained 5 items and is modified to fit the context of this study as shown in Table 3 .

\section{User Satisfaction}

It can be experienced in a variety of situations and connected to system. It is highly personal assessment that is greatly affected by user expectations. The measurement of user satisfaction construct contained 5 items and modified to the context of this study as shown in Table 3.

Attribute of Usability

In this area, Human computer Interaction (HCI) using rehabilitation technology device is used to track patient progress system. It attempted to bridge the gap between human's goals and the system. This is done by taking into account some of the issues in Integrated 
Multiple Ankle Technology Device (IMATD) by adopting practical techniques to observe human behavior and observe their performance. The measurement for attribute of usability construct contained 5 item and modified to the context of this study as shown in Table 4

Table 1 Perceived of usefulness item

$\begin{array}{lll}\text { Construct } & \text { Operational Definition } & \text { Measured Items }\end{array}$

Perceived of Usefulness
Perceived of usefulness is a feeling that physical therapist hold toward the improvement in tracking patient condition by using Integrated Multiple Ankle Technology Device (IMATD)
PU1: Integrated Multiple Ankle Technology Device (IMATD) on patient of feeling that doctors and progress system will enable usefulness physical therapist hold the doctor and physical therapist toward the improvement to get the information of the patient quickly

PU2: The Integrated Multiple Ankle Technology Device (IMATD) on patient condition by using progress system allows the doctor to follow up rehabilitation device tracking technology the patient condition from outside of the hospital

PU3: Integrated Multiple Ankle Technology Device (IMATD) on patient progress system is useful in the rapid retrieval of information from the patient

PU4: Integrated Multiple Ankle Technology Device (IMATD) on patient progress system will save the time of physical therapist. PU5: Using Integrated Multiple Ankle Technology Device (IMATD) would improve my tracking patient condition performance.

Table 2 Perceived ease of use items

Construct

Perceived Ease of Use
Operational Definition

Measured Items
Perceived Ease of use refers to a level of easiness that physical therapist feel when using Integrated Multiple Ankle Technology Device (IMATD) tracking on patient progress system
EU1: Learning to operate Integrated Multiple Ankle Technology Device (IMATD) on patient progress system would be ease for me EU2: I would find it easy get Integrated Multiple Ankle Technology Device (IMATD) tracking on patient progress system to do what I want it to do.

EU3: My interaction with Integrated Multiple Ankle Technology Device (IMATD) tracking on patient progress system would be clear and understandable.

EU4: I would find Integrated Multiple Ankle Technology Device (IMATD) tracking on patient progress system to be flexible to interact

EU5: It would be easy for me to become skillful at using Integrated Multiple Ankle Technology Device (IMATD) tracking on patient progress

Table 3 User satisfaction items

\begin{tabular}{lll} 
& \multicolumn{1}{c}{ Operational Definition } & \multicolumn{1}{c}{ Measured Items } \\
\hline User Satisfaction & $\begin{array}{l}\text { User satisfaction refers to a level of } \\
\text { satisfying that physical therapist of using } \\
\text { Integrated Multiple Ankle Technology } \\
\text { Device (IMATD) on patient progress } \\
\text { system }\end{array}$ & $\begin{array}{l}\text { Technology Device (IMATD) tracking on patient progress system } \\
\text { TS2: I feery confident in using the Integrated Multiple Ankle } \\
\text { US3: I found it easy to share information about the patient condition } \\
\text { using Integrated Multiple Ankle Technology Device (IMATD) } \\
\text { tracking on patient progress. }\end{array}$ \\
& $\begin{array}{l}\text { US4: I can accomplish the task quickly using this procedure } \\
\text { US5: I believe that from using Integrated Multiple Ankle } \\
\text { Technology Device (IMATD) tracking on patient progress system } \\
\text { will increase the quality of health care industry }\end{array}$
\end{tabular}


Table 4 Attribute of usability items

$\begin{array}{lll}\text { Construct } & \text { Operational Definition } & \text { Measured Items }\end{array}$

Attribute of Usability
Attribute of usability shows up potential issues in the rehabilitation technology device on patient progress system. The usability helps to get feedback on what is or isn't working and have a much broader understanding of what users are doing and how they interact with the system , system by progress using Integrated Multiple Ankle Technology Device (IMATD).
AU1: It easy to interact with Integrated Multiple Ankle Technology Device (IMATD) tracking on patient progress

AU2: The procedure through Integrated Multiple Ankle Technology Device (IMATD) on patient

AU3: I found it easy to decide which the case need to be AU4: I found the various functions in this system were well integrate

AU5: I think that I would like to use this system always

\subsection{CONCLUSION}

Therefore there is an eminent need to investigate the current position on the acceptance of these rehabilitation technology device applications that are tailored to track patients' condition and storing patient's information. We proposed Technology Acceptance Model (TAM) based on the literature review that aims to evaluate and investigate usability test for Perceived of usefulness, Perceived Ease of Use, User Satisfaction and Attribute of usability. These attributes are important on evaluation of Rehabilitation technology device on patients' progress system to assess Integrated Multiple Ankle Technology Device (IMATD) system to be useful for the users.

\section{References}

Ajzen, I. and M. Fishbein, (1980). Understanding Attitudes and Predicting Social Behavior. 1st Edn., Prentice Hall, Englewood Cliffs, ISBN-10:0139364439, 278. Abu-Dalbouh, H. M. (2013). A Questionnaire Approach Based On The Technology Acceptance Model For Mobile Tracking On Patient Progress Applications. Journal of Computer Science, 9(6), 763-770.

Al-Omari, A.I., K. Jaber and A. Al-Omari, (2008). Modified Ratio-Type Estimators Of The Mean Usingextreme Ranked Set Sampling. J. Math. Stat., 4: 150-155. DOI: 10.3844/jmssp.2008.150.155

Ammenwerth, E., F. Ehlers, U. Kutscha, A. Kutscha And R. Eichstadter et al., (2002). Supporting Patient Careby Using Innovative Information Technology. Disease Manage. Health Outcomes, 10: 479-487. DOI:10.2165/00115677-200210080-00004

Ajzen, I. (1991). The Theory Of Planned Behavior. Organizational Behavior And Human Decision Processes, 50(2), 179-211.

Burton-Jones, A. and G.S. Hubona, (2005). Individual Differences And Usage Behavior: Revisiting Atechnology Acceptance Model Assumption. DatabaseAdv. Inform., 36: 58-77. DOI: $10.1145 / 1066149.1066155$

Rubin, A. Babbie. (2001). Research Methods for Social Workers (4th ed.). Belmont.Blaikie, N. (2007). Approaches To Social Enquiry: Advancing Knowledge. Polity.

Chau, P.Y.K. And P.J.H. Hu, (2001). Information Technology Acceptance By Individual Professionals: A Model Comparison Approach. Decision Sci., 32:699-719. DOI: $10.1111 / j .1540-5915.2001 . t b 00978 . x$

Chau, P.Y.K. and P.J.H. Hu, (2002a). Investigating Healthcare Professionals' Decisions To Accept Telemedicine Technology: An Empirical Test Of Competing Theories. Inform. Manage., 39: 297-311. DOI: 10.1016/S0378-7206(01)00098-2

Chau, P.Y.K. and P.J.H. Hu, (2002b). Examining a Model Of Information Technology Acceptance By Individual Professionals: An Exploratory Study. J. Manage.Inform. Syst., 18: 297-311.

Chismar, W.G. and S. Wiley-Patton, (2003). Does The Extended Technology Acceptance Model Apply To Physicians. Proceedings of the 36th Annual Hawaii International Conference on System Sciences, Jan. 6-9, IEEE Xplore Press, 160-167. DOI:10.1109/HICSS.2003.1174354

Chomeya, R., (2010). Quality of Psychology Test Between Likert Scale 5 And 6 Points. J. Soc. Sci., 6: 399-403.DOI: 10.3844/jssp.2010.399.403

Creswell, J.W., (2011). Educational Research: Planning, Conducting and Evaluating Quantitative and Qualitative Research. 4th Edn., Pearson Education, Boston, ISBN10: 0132613948,650

Davis, F.D. and V. Venkatesh, (2004). Toward Preprototype User Acceptance Testing Of New Information Systems: Implications For Software Project Management. IEEE Trans. Eng. Manage., 51:31-46.

Davis, F.D., (1989). Perceived Usefulness, Perceived Easeof Use And User Acceptance Of Information Technology. MIS Q., 13: 319-340. DOI: 10.2307/249008

Davis, F.D., (1993). User Acceptance Of Information Technology: System Characteristics, User Perceptions And Behavioral Impacts. Int. J. Man-Mach. Stud., 38: 475487. DOI: $10.1006 / \mathrm{imms} .1993 .1022$

Davis, F.D., R.P. Bagozzi and P.R. Warshaw, (1989). User Acceptance Of Computer Technology: A Comparisonof Two Theoretical Models. Manage. Sci., 35: 9821003. DOI: $10.1287 / \mathrm{mnsc} .35 .8 .982$

Denzin, N. K., \& Lincoln, Y. S. (1994). Handbook of Qualitative Research. Sage publications, inc.

Fishbein, M. and I. Ajzen, (1975). Belief, Attitude, Intention and Behavior: An Introduction to Theory and Research. 1st Edn., Addison-Wesley, Reading, Mass., ISBN10: $0201020890,578$.

Grimshaw, JM., R.E. Thomas, G. MacLennan, C. Fraserand C.R. Ramsay et al., (2004). Effectiveness And Efficiency Of Guideline Dissemination And Implementation Strategies. Health Technol. Assess, 8, 1-73. PMID: 14960256

Greene, J. C., \&Caracelli, V. J. (1997). Advances In Mixed-Method Evaluation: The Challenges And Benefits Of Integrating Diverse Paradigms (No. 74).

Jossey-Bass., Jemain, A.A., A. Al-Omari and K. Ibrahim, (2007). Multistage Median Ranked Set Sampling For Estimating The Population Median. J. Math. Stat., 3:5864.

Kanaan, R.K., (2009). Making Sense Of E-Government Implementation In Jordan: A Qualitative Investigation. PhD Thesis, Center of Computing and Social Responsibility. De Montfort University, Leicester.

King, G., Keohane, R. O., \&Verba, S. (1994). Designing Social Inquiry: Scientific Inference In Qualitative Research. Princeton University Press.

Neuman, S. B., Copple, C., \&Bredekamp, S. (2000). Learning to Read And Write: Developmentally Appropriate Practices For Young Children. National Association for the Education of Young Children, 1509 16th Street, NW, Washington, DC (Stock no. 161, \$12). 
Sundaravej, T. (2010). Empirical Validation Of Unified Theory Of Acceptance And Use Of Technology Model. Journal of Global Information Technology Management, 13(1), 5-27

Maciejasz, P., Eschweiler, J., Gerlach-Hahn, K., Jansen-Troy, A., \&Leonhardt, S. (2014). A Survey On Robotic Devices For Upper Limb Rehabilitation. Journal Ofneuroengineering And Rehabilitation, 11(1), 3.

Tasir, Z., Abour, K. M. E. A., Halim, N. D. A., \& Harun, J. (2012). Relationship Between Teachers' ICT Competency, Confidence Level, And Satisfaction Toward ICT Training Programmes: A Case Study Among Postgraduate Students. TOJET: The Turkish Online Journal of Educational Technology, 11(1). 